EPJ Web of Conferences 40, 12001 (2013)

DOI: $10.1051 /$ epjconf/20134012001

(C) Owned by the authors, published by EDP Sciences, 2013

\title{
The disordered local moment approach to the spin-disorder resistivity of metallic ferromagnets
}

\author{
J. Kudrnovskýl,a, , V. Drchal ${ }^{1}$, I. Turek ${ }^{2}$, S. Khmelevskyi ${ }^{3}$, J. K. Glasbrenner ${ }^{4}$, and K. D. Belashchenko ${ }^{4}$ \\ 1 Institute of Physics AS CR, Na Slovance 2, CZ-182 21, Praha 8, Czech Republic \\ 2 Department of Condensed Matter Physics, Charles University, CZ-121 16, Praha 2, Czech Republic \\ 3 Institute of Applied Physics, Vienna University of Technology, A-1020, Vienna, Austria \\ ${ }^{4}$ Department of Physics and Astronomy and Nebraska Center for Materials and Nanoscience, University of Nebraska, \\ Lincoln, Nebraska 68588, USA
}

\begin{abstract}
The spin-disorder resistivity (SDR) of transition metal ferromagnets (Fe, Co, Ni), rare-earth ferromagnet $\mathrm{Gd}$, and $\mathrm{Ni}_{2} \mathrm{MnSn}$ Heusler alloy is determined from first principles. We identify the SDR at the Curie temperature with the residual resistivity of the corresponding system evaluated in the framework of the disordered local moment (DLM) model which has the zero spin-spin correlation function. The underlying electronic structure is determined in the framework of the tight-binding linear muffin-tin orbital method which employs the coherent potential approximation (CPA) to describe the DLM state. The DLM fixed-spin moment approach is used in the case when the DLM moment collapses. The electronic structure of hcp-Gd is determined using both the open core and LDA+U approaches. The Kubo-Greenwood approach is used to estimate the resistivity. For bcc-Fe and $\mathrm{Ni}_{2} \mathrm{MnSn}$ alloy we shall also estimate the temperature-dependent of resistivity below the Curie temperature using a semiempirical approach. Calculations are compared with the supercell Landauer-Büttiker approach developed recently as well as with available experimental data and overall good agreement is obtained.
\end{abstract}

\section{Introduction}

Temperature dependence of the resistivity is one of the basic properties of metallic systems. In normal metals and alloys without an external magnetic field are two dominant mechanisms contributing to the resistivity, namely the residual resistivity due to the scattering of conduction electrons on impurities and other structural defects, and the resistivity due to phonon scattering. In ferromagnetic metals there is an additional scattering mechanism due to magnetic fluctuations, which usually reach their maximum close to the Curie temperature $\left(T_{c}\right)[1,2]$. The latter, spindisorder part of the resistivity (SDR) is the subject of this paper. The resistivity due to phonon scattering is well understood [3] and it depends linearly on temperature $T$ above the Debye temperature.

On the other hand, first-principles calculations of SDR have not been attempted until recently. Theoretical treatment $[4,5]$ based on the $s$ - $d$-model Hamiltonian predicts a quadratic temperature-dependence for low temperatures, a constant, temperature-independent resistivity above $T_{c}$, and a $\rho \propto\left[1-M^{2}(T) / M_{0}^{2}\right]$ behavior at intermediate temperatures. SDR can also depend on magnetic short-range order, particularly in the critical region around $T_{c}$ [6]. The saturated magnetic resistivity above $T_{c}$ corresponds to the limit of vanishing spin-spin correlations. It is often extracted from experiment [7] by extrapolation of the phonon contribution to $T=0$ and subtracting the residual resistivity.

Quantitative description of the SDR from first-principles calculations requires a consistent averaging procedure. One

\footnotetext{
a e-mail: kudrnov@fzu.cz
}

option is to perform a direct averaging of the LandauerBüttiker (LB) conductance over spin configurations in supercells. This has been done for bcc-Fe and fcc-Ni $[8,9]$ and recently also for heavy rare-earth metals [10]. Another option is to use the disordered local moment (DLM) approach [11] which approximates the paramagnetic state as an uncorrelated ensemble of randomly oriented spins and solves the electronic structure problem in the coherent potential approximation (CPA). The Kubo-Greenwood linear response calculation, with proper inclusion of vertex corrections, can then be performed $[12,13]$. A semi-empirical approach [14] to calculate the SDR was implemented by assuming a quadratic temperature dependence and calculating the parameters from first principles.

In this paper the SDR is calculated using the DLM method and the standard linear response technique applied in the bulk unit cell with the inclusion of vertex corrections. We consider the transition metals bcc-Fe and fcc$\mathrm{Ni}$, the rare-earth hcp-Gd, and the Heusler alloy $\mathrm{Ni}_{2} \mathrm{MnSn}$. The results are compared with experiment and with firstprinciples calculations using direct averaging over spindisordered supercells. The coefficient of the semi-empirical $T^{2}$ term for the total resistivity is also calculated for bcc-Fe and $\mathrm{Ni}_{2} \mathrm{MnSn}$.

\section{Formalism and computational details}

The electronic structure calculations were performed using the scalar-relativistic tight-binding linear muffin-tin orbital (TB-LMTO) scheme [15] and the local density approximation (LDA) [16]. The effect of disorder (the DLM model) is described by the CPA formulated in the framework of

This is an Open Access article distributed under the terms of the Creative Commons Attribution License 2.0, which permits unrestricted use, distribution, and reproduction in any medium, provided the original work is properly cited. 
Table 1. Calculated SDR $\left(\rho_{\mathrm{SDR}}\right)$ for bcc-Fe in the KG-DLM approach are compared with a related theoretical supercell LBapproach $[8,9]$ as well as with the experimental value [7]. We also present the magnetic moments in the ferromagnetic phase $\left(M_{\mathrm{tot}}^{\mathrm{FM}}\right)$ and in the DLM phase $\left(M_{\mathrm{tot}}^{\mathrm{DLM}}\right)$ when available. The experimental lattice constant of bcc-Fe was used in calculations.

\begin{tabular}{|c|c|c|c|}
\hline & \multicolumn{3}{|c|}{ Method } \\
\cline { 2 - 4 } & KG-DLM & supercell LB & exper \\
\hline$M_{\text {tot }}^{\mathrm{FM}}\left(\mu_{\mathrm{B}}\right)$ & 2.18 & 2.22 & 2.18 \\
$M_{\text {tot }}^{\mathrm{DLM}}\left(\mu_{\mathrm{B}}\right)$ & 2.06 & - & - \\
\hline$\rho_{\mathrm{SDR}}(\mu \Omega \mathrm{cm})$ & 71.5 & 85 & 80 \\
\hline
\end{tabular}

the TB-LMTO Green's function method [17]. The same atomic sphere radius was used for all the constituent atoms in the case of Heusler alloys, and lattice constants were taken from experiment.

In fcc-Ni, the DLM moment collapses to zero while the moment in a real material is expected to persist due to longitudinal spin fluctuations. [18,19] In this case we employ the fixed-spin moment (FSM) approach [20] and treat the local magnetic moment as an adjustable parameter to recover the experimental value of the SDR [9]. In the case of hcp-Gd we used both the open-core and the LDA+U approaches [21].

The residual resistivity is then determined by the linearresponse theory as formulated in the framework of the TBLMTO-CPA method using the Kubo-Greenwood (KG) formula [22] applied to the DLM state (KG-DLM), including vertex corrections [13].

\section{Results and discussion}

In this section we present results for the SDR of transitionmetal ferromagnets $\mathrm{Fe}$ and $\mathrm{Ni}$, the rare-earth gadolinium, and $\mathrm{Ni}_{2} \mathrm{MnSn}$ Heusler alloy.

\subsection{Transition metal ferromagnets: bcc-Fe and fcc-Ni}

We have performed KG-DLM calculations in the framework of the spdf basis set. The results are summarized in Table 1. The magnitude of the ferromagnetic local Femoment is in a good agreement with the experiment as well as with the corresponding DLM value in agreement with previous studies [11]. The KG-DLM and supercell LB results for SDR agree both well with experiment. The small differences between both theoretical approaches are mainly due to the small difference in the local moments. The effect of vertex corrections in bulk KG calculations (KG-DLM) is only a few percent.

In fcc-Ni the static local moment in the DLM state is unstable, and the calculation of SDR can not proceed in the usual way. However, electrons are still expected to be scattered by fluctuating local moments $[18,19]$. In previous supercell LB calculations [8] the local moment in fcc-Ni was used as an adjustable parameter, and it was found that agreement with experiment requires the local moment of about $0.35 \mu_{B}$. Here we proceed in the same way without attempting to evaluate the effective local moment in the paramagnetic state [19] directly. The atomic potentials are
Table 2. Calculated SDR $\left(\rho_{\text {SDR }}\right)$ for ferromagnetic fcc-Ni in the KG-DLM approach is compared with the results of the supercell LB approach [8,9]. The experimental value is $15 \mu \Omega \mathrm{cm}[7,23]$. Calculated resistivities are presented as a function of the effective Ni-local moment $M_{\text {eff }}$. Results are shown for the $s p d f$-basis. In the case of the LB approach we also show theoretical errorbars [9]. The experimental lattice constant of fcc-Ni was used.

\begin{tabular}{|c|c|c|}
\hline Method & $M_{\text {eff }}\left(\mu_{\mathrm{B}}\right)$ & $\rho_{\text {SDR }}(\mu \Omega \mathrm{cm})$ \\
\hline KG-DLM & 0.3 & 10.2 \\
& 0.45 & 19.7 \\
& 0.6 & 29.7 \\
\hline supercell LB & 0.3 & $12 \pm 0.3$ \\
& 0.4 & $18 \pm 0.4$ \\
& 0.5 & $23 \pm 0.5$ \\
& 0.66 & $29 \pm 0.6$ \\
\hline
\end{tabular}

prepared using the FSM method by constraining the Nilocal moment to several values: $0.6,0.45$, and $0.3 \mu_{\mathrm{B}}$. (The self-consistent value in the ferromagnetic state is $0.604 \mu_{\mathrm{B}}$ for the $\operatorname{spdf}$ basis set.)

The results for fcc-Ni are summarized in Table 2, which also includes the supercell LB results [9] and the experimental value. The KG-DLM calculations agree well with the LB results for all chosen values of the local moment. The experimental SDR is reproduced using a local moment value close to $0.35 \mu_{\mathrm{B}}$.

\subsection{Rare earth hcp Gadolinium}

The rare earth metals are interesting another class of magnetic metals in which $4 f$ electrons responsible for their large local moments are much more localized as $3 d$ electrons in the transition metal ferromagnets. Their orbital moment are not quenched in general (irrelevant for $\mathrm{Gd}$ ). This may indicate some limitations in evaluation of the SDR in which a classical spin rotation model used above is adopted. We shall consider in some detail the case of ferromagnetic hcp-Gd for which the experimental data are available $[7,24]$. If spin-orbit is strong enough, the $f d$ model predicts that the SDR is proportional to $(J+1) J$ where $J$ is a fixed angular momentum. On the contrary, if the spin-orbit coupling is small to relevant energy scales, the SDR should be proportional to $(S+1) S$. The relevant energy scales (spin-orbit splitting, exchange splitting, the bandwidth) are roughly of the same order of magnitude in hcp-Gd and this complicates the physics.

We will determine the SDR in hcp-Gd using approach developed above for transition metal ferromagnets but its electronic structure should reflects the localized character of $4 f$ electrons properly which are not well described by the LDA. We employ two approaches, namely (i) the treatment of the $4 f$ orbitals as fully localized by excluding them from valence states and filling them in accordance with Hund's rule (the open core approach), and (ii) the approximate treatment of electron correlations in narrow $4 f$ bands in the framework of the LDA+U method [21]. In the former approach are $4 f$ electrons explicitly excluded from the transport while in the latter approach not but their influence is small. The atomic potentials were constructed from the ferromagnetic state [10] and results are presented in Table 3. 
Table 3. Calculated theoretical SDR (in $\mu \Omega \mathrm{cm}$ ) for in-plane and c-axis cases in the hcp-Gd as obtained using the KG-DLM and supercell LB approaches. The experimetal results are also shown. The experimental lattice constant was used.

\begin{tabular}{|c|c|c|c|}
\hline \multirow{2}{*}{ SDR type } & \multicolumn{3}{|c|}{ SDR in hcp Gadolinium } \\
\cline { 2 - 4 } & KG-DLM & supercell LB & Exp \\
\hline In-plane SDR & 59.1 & 58.9 & 105 \\
\hline c-axis SDR & 40.2 & 42.0 & 95 \\
\hline
\end{tabular}

The SDR determined by the KG-DLM and supercell LB approaches obtained in the framework of the open core approach agree very well each to other. The calculated inplane resistivity is greater as compared to the $c$-axis resistivity in agreement with experiment suggesting a proper description of the electronic structure anisotropy in the firstprinciples calculations. On the other hand, the absolute values of in-plane and $c$-axis resistivities are significantly underestimated as compared to the experiment.

The localized character of $4 f$-states of Gd suggests the relevance of their quantum character. We have scaled the SDR components by a factor $(S+1) / S$ (in Gd it is the same scaling by a factor $(J+1) / J$ ) to account for the quantum correction effects missing in the present approach. As a result the SDR was enhanced to about $75 \mu \Omega \mathrm{cm}$ and $60 \mu \Omega \mathrm{cm}$ for the in-plane and c-axis. Despite of obvious improvement, the agreement between the theory and experiment is still worse as compared to the transition metal ferromagnets or Heusler alloys (see below). In the opencore approach the resistivity depends of the exchange parameter $\delta$ of $5 d$-bands: the larger splitting represents large disorder in the DLM-state. We have tested this by performing the LDA+U calculations applied to $5 d$-band so that calculated $d$-moments increased from its LDA value $0.68 \mu_{\mathrm{B}}$ to $0.76 \mu_{\mathrm{B}}$. The calculated SDR values were $77 \mu \Omega \mathrm{cm}$ and $60 \mu \Omega \mathrm{cm}$ for the in-plane and c-axis. The reader interested in more details and also to results for other members of the Gd-Tm family is referred to a recent paper [10].

\subsection{Heusler alloy $\mathrm{Ni}_{2} \mathrm{MnSn}$}

Heusler alloys are another magnetic metals for which experimental data are available in the literature. These alloys have $\mathrm{L} 2_{1}$-structure formed by four interpenetrating fcc sublattices mutually shifted along the body-diagonal with the sublattice occupation Ni-Mn-Ni-Sn for $\mathrm{Ni}_{2} \mathrm{MnSn}$. We employ the $s p d f$ basis and the DLM model for the Mn sublattice. Small induced magnetic moments on the $\mathrm{Ni}$ and $\mathrm{Sn}$ atoms in the ferromagnetic state collapse in the DLM state, and their effect is neglected here. The experiment also shows negligible residual resistivity at $T=0 \mathrm{~K}$ for $\mathrm{Ni}_{2} \mathrm{MnSn}$ [25] which simplifies comparison with the calculated value. On the contrary, e.g. in $\mathrm{Ni}_{2} \mathrm{MnSb}$ alloy, there is a large residual resistivity due to alloy disorder present even in the stoichiometric alloy [25]. The calculated results using the KG-DLM and supercell LB approach are compared in Table 4. The calculated SDR values obtained by the KG-DLM approach agree well with both the experiment and results of the supercell LB approach. We note that the spin disorder is almost fully concentrated on the Mn-sublattice and the moments have predominantly $d$ character similarly like in transition metal ferromagnets.
Table 4. Calculated theoretical SDR in Heusler alloy $\mathrm{Ni}_{2} \mathrm{MnSn}$ in the KG-DLM approach is compared with corresponding experimental data [8] and related supercell LB approach. The DLM is limited to the Mn-sublattice. The experimental lattice constant was used.

\begin{tabular}{|c|c|c|c|}
\hline \multirow{2}{*}{ Alloy } & \multicolumn{3}{|c|}{ SDR in Heusler alloys (in $\mu \Omega \mathrm{cm}$ ) } \\
\cline { 2 - 4 } & KG-DLM & supercel LB & experiment \\
\hline $\mathrm{Ni}_{2} \mathrm{MnSn}$ & 50.4 & $47.7 \pm 0.4$ & 46.6 \\
\hline
\end{tabular}

\subsection{Resistivity of bcc-Fe and $\mathrm{Ni}_{2} \mathrm{MnSn}$ below $T_{c}$}

The determination of the $T$-dependent resistivity below $T_{c}$ is a difficult problem not fully solved yet. A direct approach to this problem can be realized in the framework of the LB approach: we construct supercells and map the real-space spin configuration as obtained e.g. from MonteCarlo simulations for a classical Heisenberg Hamiltonian at the specified temperature $T$. The Monte-Carlo method is particularly useful as it can be also used to include magnetic short-range order (MSRO) effects [9] which are otherwise difficult to include in analytic theories [5,6]. A simpler approach can be used for systems in which the MSRO effects are weak, like, e.g., in some Heusler alloys [14] or bcc-Fe. For example, an empirical expression $\rho(T)=$ $\rho_{o}+A T+C T^{2}$ is obeyed reasonably well in a number of Heusler alloys [25]. It should be pointed out that this is an empirical fact and not a rigorous theory, and that in other cases, e.g. for rare-earth metals the above expression is invalid.

However, for systems where this relation is observed one can approximate the SDR contribution as $\rho_{\mathrm{SDR}}(T)=$ $C T^{2}$, where the constant $C=\rho\left(T_{c}\right) / T_{c}^{2}$. If one identifies $\rho\left(T_{c}\right)$ with the SDR as calculated above in the framework of the KG-DLM approach, it is possible to estimate the $T$-dependence of the resistivity also below $T_{c}$. Because $T_{c}$ can be determined from the Heisenberg model constructed from the same electronic structure as used for the SDR calculations, the coefficient $C$ unifies in a single number relevant transport and thermodynamical characteristics of the system and its estimate is thus a test of internal consistency of the present approach. Such a program was successfully tested for the case of $\mathrm{Ni}_{2} \mathrm{MnSn}$ and $\mathrm{Pd}_{2} \mathrm{MnSn}$ Heusler alloys (see [14] for details) and here we wish to check it for bcc-Fe ferromagnet.

Using the same electronic structure as used in transport calculations (DLM state, $s p d f$-basis set) we have constructed the corresponding Heisenberg Hamilonian [26] for the estimate of $T_{c}$. The Curie temperature was determined in the framework of the random-phase approximation and we obtained $T_{c}=1105 \mathrm{~K}$ (the experiment is $1040 \mathrm{~K}$ ). We have estimated also the constant $C$ using the calculated SDR in the KG-DLM (see Table 1) and obtained $C=0.586 \times$ $10^{-4} \mu \Omega \mathrm{cm} / \mathrm{K}^{2}$. To compare with experiment, we used the tabulated electrical resistivity data for Fe [7] from which we have extracted the phonon contribution. The residual resistivity of pure bcc-Fe is negligible (of order $0.1 \mu \Omega \mathrm{cm}$ ).

The result is shown in Fig. 1. We mention an overall good agreement between the present simple theory and experiment below $T_{c}$ which, in turn, justifies the first principles estimate of the coefficient $C$ in this case. It should be noted that similarly good agreement was obtained also for $T$-dependent resistivity of $\mathrm{Ni}_{2} \mathrm{MnSn}$ below $T_{c}$. Some deviations were due to the offset between calculated (322 K) 


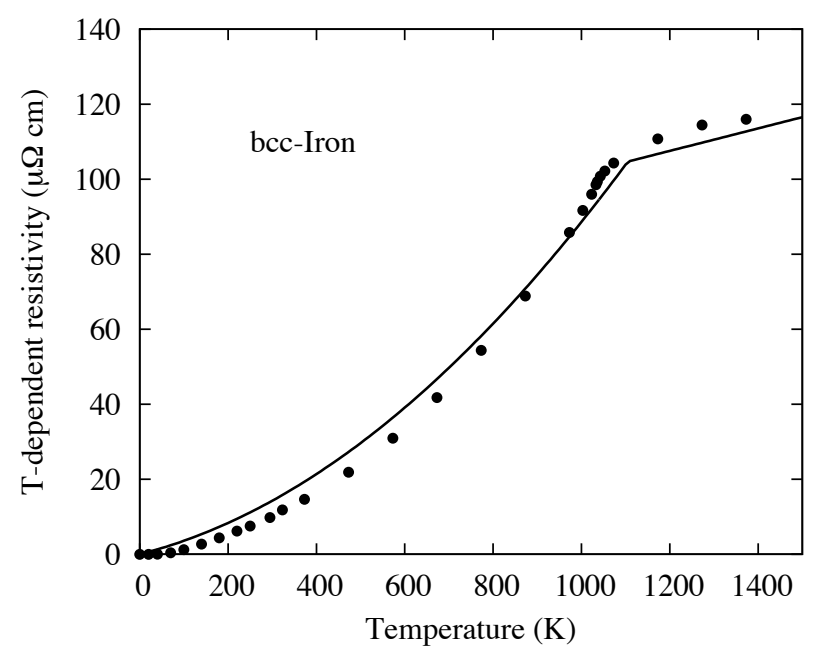

Fig. 1. Temperature dependence of the resistivity of bcc-Fe below $T_{c}$ assuming empirical $T^{2}$-dependence (see text for details). The theory (full line) is compared with the experiment (bullets) [7].

and experimental (340 K) Curie temperatures [14]. In both cases, however, with the exception of very low temperatures, the contribution due to spin-disorder scattering dominated that coming from scattering on phonons.

\section{Conclusions}

We have presented a simple theory of the paramagnetic spin-disorder resistivity based on the disordered local moment model combined with the Kubo-Greenwood linearresponse technique and applied it to magnetic transition metals, to the hcp Gadolinium, and to the $\mathrm{Ni}_{2} \mathrm{MnSn}$ Heusler alloy. The results agree reasonably well with experimental data and with the results of the supercell LandauerBuittiker approach in all systems considered. The case of $\mathrm{Ni}$ requires a special approach in which the FSM-DLM method is used. Present results, in an agreement with a recent study, [9] indicate an interesting relation between the SDR and the local Ni moments in the magnetically disordered state, where the local moment is induced by the longitudinal spin fluctuations [19]. The agreement obtained for hcp-Gd is much less satisfactory as the theoretical calculations underestimate the SDR values but reproduce correctly the larger in-plane value as compared to the c-axis value. This indicates some limitations of classical spindisorder model and necessity to include quantum corrections (e.g. a correction factor $(S+1) / S$ slightly improves agreement with the experiment). Finally, we have shown that in particular cases it is also possible to estimate the magnetic contribution to the resistivity below $T_{c}$ in systems with small magnetic short-range order at $T_{c}$ by assuming its quadratic dependence on $T$ but this conclusion has to be taken with a caution.

J.K., V.D., and I.T. acknowledge support of the Czech Science Foundation (P204/12/0692). The work at UNL was supported by the NSF through Grant No. DMR- 1005642 and Nebraska MRSEC (Grant No. DMR-0820521). K.D.B. is a Cottrell Scholar of the Research Corporation.

\section{References}

1. B. R. Coles, Adv. Phys. 7, (1958) 40.

2. N. F. Mott, Adv. Phys. 13, (1964) 325.

3. J.M. Ziman, Electrons and Phonons (Oxford, 1960).

4. T. Kasuya, Prog. Theor. Phys. 16, (1956) 58.

5. P.G. de Gennes and J. Friedel, J. Phys. Chem. Solids 4, (1958) 71.

6. M.E. Fisher and J.S. Langer, Phys. Rev. Lett. 20, (1968) 665.

7. J. Bass and K.H. Fischer in Landolt-Börnstein New Series Group III, Vol. 15a, K.-H. Hellwege and J.L. Olsen (Eds.) (Springer, Berlin, 1982) p. 1.

8. A. Wysocki, K.D. Belashchenko, and J.P. Velev, J. Appl. Phys. 101, (2007) 09G506.

9. A. Wysocki, R.F. Sabirianov, M. van Schilfgaarde, and K.D. Belashchenko, Phys. Rev. B 80, (2008) 224423.

10. J.K. Glasbrenner, K.D. Belashchenko, J. Kudrnovský, V. Drchal, S. Khmelevskyi, and I. Turek, Phys. Rev. B 85, (2012) 214405.

11. B.L. Gyorffy, A.J. Pindor, J. Staunton, G.M. Stocks, and H. Winter, J. Phys. F: Metal Phys. 15, (1985) 1337.

12. V. Drchal, J. Kudrnovský, P. Bruno, P.H. Dederichs, I. Turek, and P. Weinberger, Phys. Rev. B 65, (2002) 214414.

13. K. Carva, I. Turek, J. Kudrnovský, and O. Bengone, Phys. Rev. B 73, (2006) 144421.

14. S.K. Bose, J. Kudrnovský, V. Drchal, and I. Turek, Phys. Rev. B 82, (2010) 174402.

15. O.K. Andersen and O. Jepsen, Phys. Rev. Lett. 53, (1984) 2571.

16. S.H. Vosko, L. Wilk, and M. Nusair, Can. J. Phys. 58, (1980) 1200.

17. I. Turek, V. Drchal, J. Kudrnovský, M. Šob, and P. Weinberger, Electronic Structure of Disordered Alloys, Surfaces and Interfaces (Kluwer, Boston, 1997); I. Turek, J. Kudrnovský and V. Drchal, in Electronic Structure and Physical Properties of Solids, edited by H. Dreyssé, Lecture Notes in Physics, Vol. 535 (Springer, Berlin, 2000), p. 349.

18. M. Uhl and J. Kübler, Phys. Rev. Lett. 77, (1996) 334.

19. A.V. Ruban, S.K. Khmelevskyi, P. Mohn, and B. Johansson, Phys. Rev. B 75, (2007) 054402.

20. V.L. Moruzzi, P.M. Marcus, K. Schwarz, and P. Mohn, Phys. Rev. B 34, (1986) 1784.

21. P. Kurz, G. Bihlmayer, and S. Blügel, J. Phys.: Condens. Matter 14, (2002) 6363.

22. I. Turek, J. Kudrnovský, V. Drchal, L. Szunyogh, and P. Weinberger, Phys. Rev. B 65, (2002) 125101.

23. R.J. Weiss and A.S. Marotta, J. Phys. Chem. Solids 9 , (1959) 302 .

24. K. Maezawa, K. Mori, K. Sato, Y. Saito, and S, Wakabayashi, J. Phys. Soc. Japan 43, (1977) 1815.

25. W.H. Schreiner, D.E. Brandao, F. Ogiba, and J.V. Kunzler, J. Phys. Chem. Solids 43, (1982) 777.

26. I. Turek, J. Kudrnovský, V. Drchal, and P. Bruno, Philos. Mag. 86, (2006) 1713. 